\title{
ANATOMÍA VEGETATIVA DE CTENITIS MELANOSTICTA (DrYOPTERIDACEAE, PTERIDOPHYTA)
}

\author{
Victoria Hernández-HernándeZ', Teresa TerRaZAS ${ }^{2,4}$ y KLaus MeHLTReter ${ }^{3}$ \\ 'Programa de Botánica, Colegio de Postgraduados, Montecillo, Edo. de México, C.P. 56230, México. \\ ${ }^{2}$ Departamento de Botánica, Instituto de Biología, Universidad Nacional Autónoma de México, \\ Apdo. Postal 70-233, México 04510, D.F., México. \\ ${ }^{3}$ Departamento de Ecología Funcional, Instituto de Ecología, A.C., Km 2.5 antigua carretera a Coatepec No. 351 , \\ Congregación El Haya, Xalapa 91070, Veracruz, México. \\ ${ }^{4}$ Autor para la correspondencia. Correo-e: tterrazas@ibiologia.unam.mx
}

\begin{abstract}
Resumen: En este estudio se investigó la anatomía de la raíz, el rizoma, el pecíolo y la lámina de Ctenitis melanosticta, y se compararon los resultados con los rasgos registrados para otros géneros filogenéticamente cercanos. La raíz es diarca con la parte interna del córtex conformada por esclereidas y células de parénquima con hifas fungales en la parte externa. El rizoma tiene nidos de esclereidas, al igual de lo que sucede en los géneros Dryopteris y Campyloneurum. Una banda cortical se encontró en el pecíolo y la lámina, similar a lo registrado en Dryopteris y otros géneros de helechos avanzados. El mesofilo de la hoja es unifacial y el lado adaxial subyacente a la epidermis presenta varios estratos de fibras, al igual que en Elaphoglossum y Thelypteris, pero esto difiere de los géneros Asplenium, Dryopteris y Polybotrya, cuyas especies tienen colénquima en el mismo sitio. La anatomía fue similar respecto a especies estudiadas de la familia Dryopteridaceae, pero hubo algunas diferencias notables. La realización de más estudios anatómicos en especies de Dryopteridaceae permitirá confirmar el valor diagnóstico de los rasgos anatómicos, tales como la ausencia de banda cortical en el rizoma, la presencia de nidos de esclereidas, el mesofilo unifacial y la presencia de banda cortical en la lámina.
\end{abstract}

Palabra clave: banda cortical, Ctenitis melanosticta, esclerénquima, mesofilo unifacial, raíz diarca, vena primaria.

\begin{abstract}
The root, rhizome, petiole and blade anatomy of Ctenitis melanosticta was studied and compared with the available information for closely related genera. Root is diarc with sclerenchyma and parenchyma cells in the cortex, with fungal hyphae exclusively in the latter. The occurrence of sclereid nests in the rhizome is shared with Dryopteris and Campyloneurum. A cortical band was present in petiole and lamina, as in Dryopteris and other genera of the most derived ferns. The lamina had unifacial mesophyll and under the adaxial epidermis there were several layers of the fiber, as described for Elaphoglossum and Thelypteris, but differed from Asplenium, Dryopteris, and Polybotrya with collenchyma. The anatomy of C. melanosticta was similar to that of most species studied of Dryopteridaceae, although with some differences. Additional anatomical studies in species of Dryopteridaceae will allow to confirm the diagnostic value of several anatomical features, such as the lack of cortical band in the rhizome, the sclereid nests, the unifacial mesophyll, and the cortical band in the lamina.
\end{abstract}

Key words: cortical band, Ctenitis melanosticta, diarc root, mid-vein, sclerenchyma, unifacial mesophyll.

$\mathbf{E}^{1}$ género Ctenitis, que junto con otros 18 géneros confo rma la familia Dryopteridaceae, está constituido por 150 especies cuyo centro de dive rsidad es la región neotrpical (Crabbe et al., 1975; Mickel y Smith, 2004). Con base en las secuencias $r b c \mathrm{~L}$, se considera que esta familia pertenece al clado de pteridofitas más avanzadas, junto con Aspleniaceae, Blechnaceae, Grammitidaceae, Lomariopsidaceae, Nephrolepidaceae, Polypodiaceae y
Thelypteridaceae (Hasebe et al., 1995). En México se presentan 21 especies de Ctenitis, de las cuales tres son endémicas: Ctenitis bullata A.R.Sm., C. chiapasensis (H.Christ) A.R.Sm. y C. ursina A.R.Sm. Las especies de Ctenitis son plantas terrestres o epipétricas que habitan bosques húmedos a altitudes de entre 50 y 1,800 m (Tryon y Tryon, 1982).

Ctenitis melanosticta (Kunze) Copel. es una especie 
cuyo estatus de conservación en México es indeterminado. En el Parque Francisco Javier Clavijero, Xalapa, Veracruz, existen poblaciones con individuos fértiles en todas las clases de tamaño y edades; además, es una especie longeva que durante la mayor parte del año tiene frondas fértiles (Hernández-Rojas, 2006). Estas particularidades hacen de Ctenitis melanosticta un objeto de estudio adecuado para realizar investigación ontogenética sobre su crecimiento y sus características anatómicas, y para utilizarlo como modelo para el estudio comparativo de otros helechos. A la fecha, los trabajos realizados con Ctenitis melanosticta se limitan a descripciones taxonómicas (Smith, 1981; Mickel y Beitel, 1988; Moran, 1995; Mickel y Smith, 2004). Esta especie se distribuye en el bosque mesófilo de montaña y en la selva alta perennifolia -sensu Miranda y HernándezX. (1963)-, en los estados de Hidalgo, Oaxaca, Puebla, Querétaro, San Luis Potosí, Tabasco y Veracruz, mientras que en Centroamérica se distribuye desde Guatemala hasta Costa Rica. Son plantas que tienen un rizoma suberecto con escamas café oscuras, lineares y margen entero. Sus frondas llegan a medir entre 100 y $130 \mathrm{~cm}$ de longitud (figura 1), el pecíolo es estramíneo, con escamas en la base, y hacia el raquis está recubierto por tricomas multicelulares. La lámina es deltada, de 2 a 3 veces pinnada-pinnatífida, de forma más o menos equilateral, con 12 ó 15 pares de pinnas (Mickel y Smith, 2004).

Los estudios que abordan la anatomía comparada de las especies de helechos avanzados son escasos y se han limitado a unas pocas especies. Por ejemplo, se ha descrito y comparado la anatomía de especies de los géneros Dyopteris (Hernández-He mández et al., 2006), Polybotrya (Moran, 1987), Polypodium (Tejero-Diez, 2005), Tectaria (Velásquez-Montes, 1983) y Thelypteris (Zavaro-Pérez et al., 1995), o bien se han realizado estudios comparativos de especies pertenecientes a más de dos géneros (Lucansky, 1981; Zlotnik-Espinosa, 1991; Umikalsom, 1992). El objetivo del presente estudio fue describir la anatomía de los órganos vegetativos de Ctenitis melanosticta y comparar sus ras gos anatómicos con los de otros géneros de lafamilia Dryopteridaceae o familias filogenéticamente cercanas.

\section{Materiales y métodos}

Se recolectaron tres individuos completos de Ctenitis melanosticta en el Parque Francisco Javier Clavijero, Xalapa, Veracruz (ejemplar de respaldo, HernándezHernández 485, depositado en CHAPA). El mat erial fresco se separó por órgano y cada uno, raíz, rizoma, pecíolo, raquis, lámina, fue cortado como se menciona a continuación. De la raíz se cortaron cilindros de $0.5 \mathrm{~cm}$ de largo; del rizoma se obtuvieron dos porciones de forma irregular, dependiendo del número de bases de los pecíolos que no se desprendieron; del pecíolo se tomaron segmentos $(1 \mathrm{~cm}$ de largo) de la parte basal, media y superior, así como segmen-

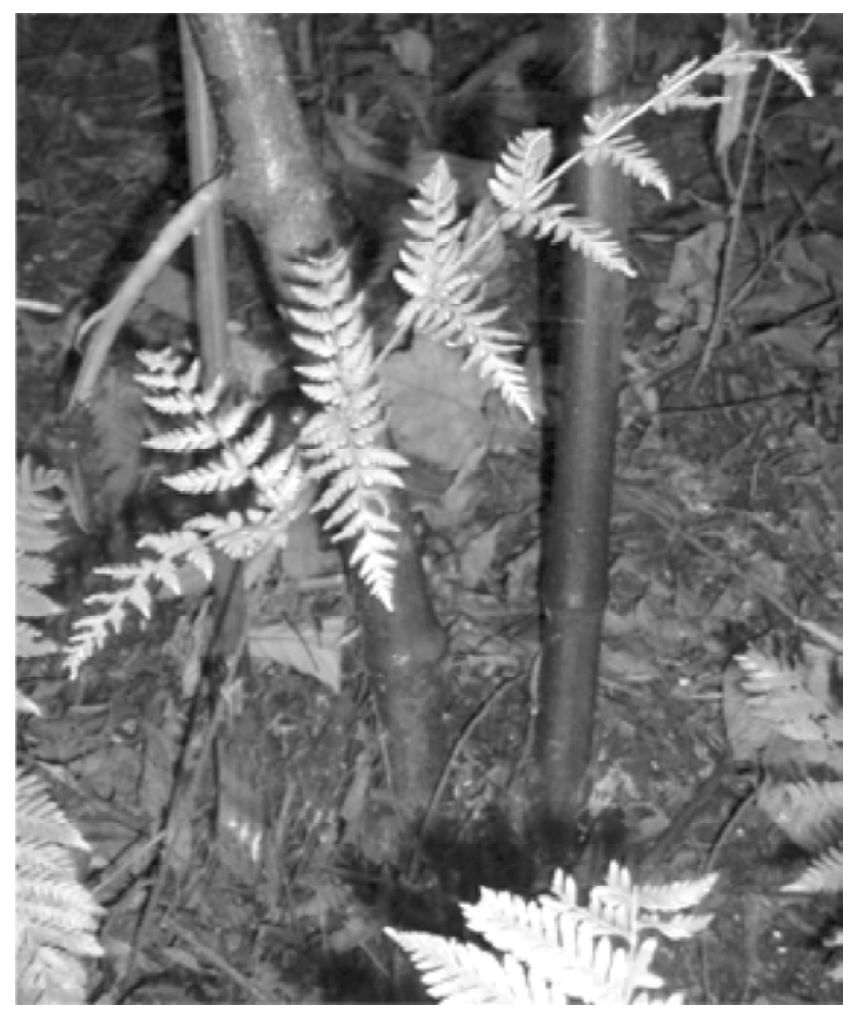

Figura 1. Planta de Ctenitis melanosticta.

tos del raquis y de la pinna (lámina) en la parte media de la fronda. Todo el material se fijó en formaldehído-ácido acético glacial-alcohol etílico (Ruzin, 1999).

Se hicieron cortes transversales del pecíolo a mano, por ser lo suficiente rígido y no requerir inclusión de ningún tipo, y la mitad de los cortes se aclararon con hipoclorito de sodio (cloro comercial al 50\%) para eliminar el contenido celular. Todos los cortes fueron deshidratados en concentraciones de alcohol etílico (50\%, 70\%, 95\%), teñidos con safranina y verde rápido, y montados en resina sintética (Ruzin, 1999). Los segmentos de raíz y lámina fueron deshidratados en un cambiador automático Leica TP1020 e incluidos en parafina por ser delgados, suaves y requerir un soporte para su corte. Una vez incluidos en parafina se hicieron cortes transversales en ambos órganos y además, cortes paradermales para la lámina, en un micrótomo rotatorio a $16-20 \mu \mathrm{m}$ de grosor. Los cortes transversales de rizoma se hicieron en un micrótomo de deslizamiento a 25$40 \mu \mathrm{m}$ de grosor; una parte de los cortes se aclararon con hipoclorito de sodio (cloro comercial al 50\%) y otra parte su sumergió en ácido clorhídrico concentrado por 15-25 min para eliminar el almidón. Todos los cortes se tiñeron con safranina y verde-rápido y se montaron en resina sintética (Ruzin, 1999). Pinnas de la base y la parte media de la fronda fueron diafanizadas siguiendo la técnica utilizada por Hernández-Hernández et al. (2006) para especies del 
género Dryopteris. Se agregó floroglucinol y $\mathrm{HCl}$ a cortes no aclarados para confirmar la presencia de lignina en rizoma y pecíolo (Ruzin, 1999). Se tomaron fotografías utilizando el programa Image Pro-Plus® versión 3.1, usando una cámara de video Hitachi KP-D51 y un microscopio Olympus BX50. La terminología empleada se basó en la literatura especializada para pteridofitas (Ogura, 1972; Lucansky, 1981; Schmid, 1982; Moran, 1987).

\section{Resultados}

Raíz. La epidermis es simple con células rectangulares y escasos tricomas multicelulares (figuras 2a, b y d). El córtex está constituido por parénquima y esclerénquima; la parte externa adyacente a la epidermis presenta de 6 a 9 estratos de células de parénquima; entre 3 y 4 estratos tienen células obliteradas radialmente y subyacentes a estos estratos se observan 4 estratos de células con citoplasma denso y con hifas fungales, de forma rectangular en el corte transversal y alargadas en el corte tangencial (figuras $2 b-d$ ). El esclerénquima se localiza entre estas células de parénquima y la estela, y se compone de 4 a 7 estratos de escl e re idas. Las escle reidas más cercanas a la estela tienen las paredes secundarias con mayor grosor (figuras $2 c$, e). La endodermis tiene células rectangulares y posee una banda de
Caspari en sus paredes radiales, sin ningún tipo de contenido celular. La estela es diarca; el metaxilema se encuentra en su parte central, el protoxilema en sus dos polos radiales, y el floema entre estos dos polos de protoxilema, al lado del metaxilema. El periciclo está formado por 1 a 2 estratos de células (figuras 2e, f); además, se observan algunas células de parénquima entre xilema y floema.

Rizoma. La epidermis es simple, con células rectangulares y cuadradas y contenido obscuro que ocluye su lumen celular (figuras 3a, b). El córtex está constituido por esclerénquima y parénquima (figuras $3 a, b)$. El esclerénquima se distribuye notablemente en dos regiones, la típica subyacente a la epidermis, donde forma más de 15 estratos de células, y los denominados nidos, dispersos en el parénquima. El esclerénquima está constituido exclusivamente por esclereidas, cuyas células tienen paredes moderadamente gruesas y están impregnadas de contenido obscuro, el cual a veces ocluye totalmente su lumen celular. Los nidos de esclereidas no presentan espacios intercelulares; además, en cortes no aclarados hay abundante contenido de color café, tanto en el lumen como en sus paredes (figuras $3 \mathrm{c}, \mathrm{d})$. Las células del parénquima son isodiamétricas y comúnmente contienen granos de almidón (figuras $3 b$, c); entre sus paredes delgadas se aprecian espacios intercelu-

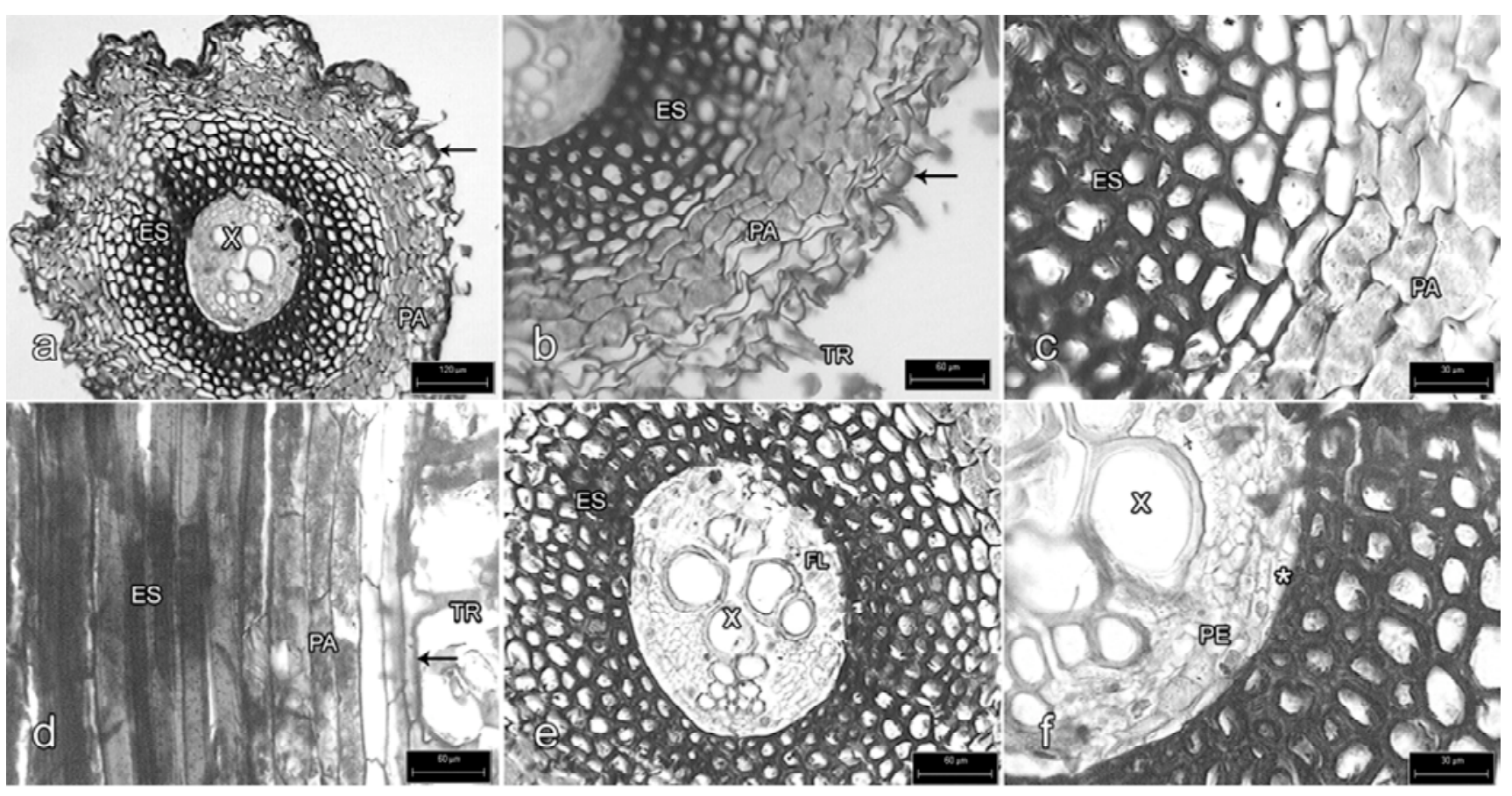

Figura 2. Raíz, cortes transversales (con excepción de d). a. Vista general de la raíz. b. Células de la epidermis, tricomas, parénquima y esclerénquima. c. Detalle de esclerénquima y parénquima. d. Corte longitudinal con tricoma, epidermis, parénquima y esclereidas. e. Raíz diarca. f. Detalle de estela y endodermis. Escalas: $\mathbf{a}=120 \mu \mathrm{m} ; \mathbf{b}, \mathbf{d}, \mathbf{e}=60 \mu \mathrm{m} ; \mathbf{c}, \mathbf{f}=30 \mu \mathrm{m}$. Clave de símbolos: * endodermis, flecha $=$ epidermis, $\mathrm{ES}=$ esclerénquima, $\mathrm{FL}=$ floema, $\mathrm{PA}=$ parénquima, $\mathrm{PE}=$ periciclo, $\mathrm{TR}=$ tricoma, $\mathrm{X}=\mathrm{xilema}$. 

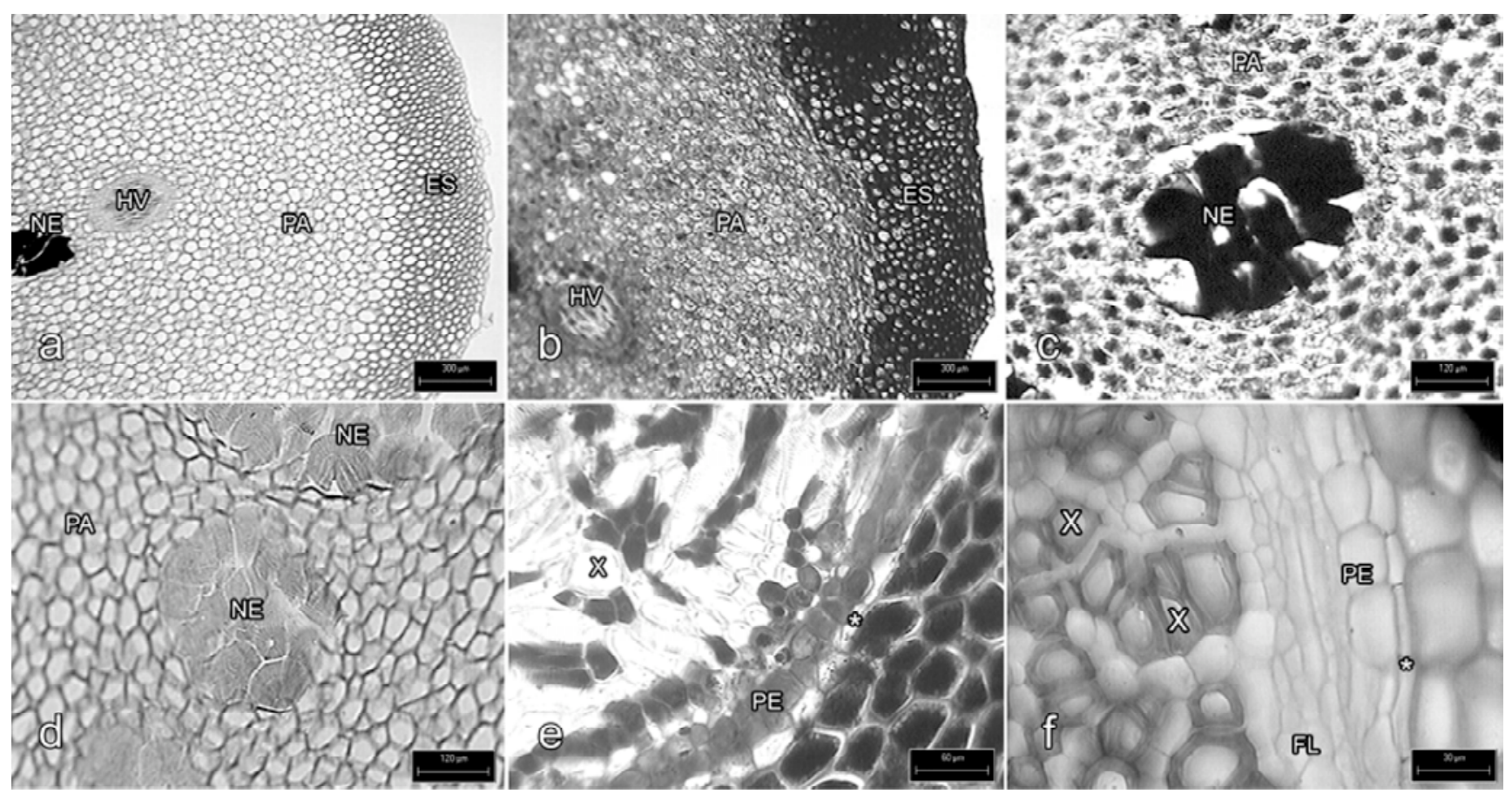

Figura 3. Rizoma, cortes transversales. a. Vista general de corte aclarado. b. Vista general de corte no aclarado. c. Nido de esclereidas no aclarado. d. Nido de esclereidas aclarado. e. Haz vascular y endodermis. f. Detalle del haz vascular. Escalas: $\mathbf{a}, \mathbf{b}=300 \mu \mathrm{m}, \mathbf{c}, \mathbf{d}=$ $120 \mu \mathrm{m}, \mathbf{e}=60 \mu \mathrm{m}, \mathbf{f}=30 \mu \mathrm{m}$. Clave de símbolos: * = endodermis, $\mathrm{ES}=$ esclerénquima, $\mathrm{FL}=$ floema, $\mathrm{HV}=\mathrm{haz}$ vascular, $\mathrm{NE}=$ nido de esclereida, $\mathrm{PA}=$ parénquima, $\mathrm{PE}=$ periciclo, $\mathrm{X}=$ xilema.

lares. La estela es una dictiostela que presenta 6 a 11 haces vasculares de fo rma ovalada o redonda. Alrededor de cada haz vascular se presenta la endodermis, con las mismas características descritas para la raíz (figuras $3 e$, f). Los haces vasculares están formados por el periciclo con 2 a 3 estratos de células, el xilema con elementos traqueales, el floema de células pequeñas, y células de parénquima entre el xilema y el floema con contenido obscuro (figuras $3 \mathrm{e}, \mathrm{f}$ ).

Pecíolo. En corte transversal, el pecíolo es cilíndrico con un surco en la cara adaxial que se extiende hacia el raquis (figura 4a). La cutícula es delgada y lisa; la epidermis es simple, con células de pared moderadamente gruesas, de forma cuadrada a rectangular y sin inclusiones en su lumen celular. El tejido fundamental está compuesto por esclerénquima y parénquima. En la parte externa subyacente a la epidermis se observa el esclerénquima, constituido por 8 a 10 estratos de fibras en la base y por 5 a 6 estratos de fibras hacia el raquis; las fibras tienen forma poligonal o circular en corte transversal y paredes gruesas (figura 4b). Subyacentes al esclerénquima están las células isodiamétricas del parénquima, con paredes delgadas y algunas veces ocluidas por granos de almidón y contenido obscuro; también se observan pequeños espacios intercelulares entre ellas. Rodeando a cada cordón vascular se encuentra una banda cortical, constituida por un estrato de células más largas que anchas hacia el centro de la traza foliar de la base del pecíolo y, en el resto de éste, las células son cuadradas con un tamaño similar en todas sus paredes; todas tienen engrosamientos en la pared periclinal interna en forma de "U" y abundante contenido color café (figuras $4 \mathrm{c}-\mathrm{f})$. Junto a la banda cortical se observa la endodermis, cuyas características son similares a las descritas para la raíz (figuras 4d, e).

En la base del pecíolo la traza foliar múltiple se compone de cinco cordones vasculares y hacia la parte superior del pecíolo su número se reduce a cuatro. El arreglo de los cordones vasculares anficribales es en forma de arco; hacia el lado adaxial se localizan los cordones de mayor tamaño y hacia el lado abaxial los de menor tamaño. En los cordones vasculares grandes, el xilema se arregla en forma de hipocampo, con la terminación del xilema arqueada hacia adentro, mientras que en los cordones vasculares pequeños el xilema se acomoda en forma circular (figuras 4d-f). En cada cordón vascular se observan los elementos traqueales con paredes lignificadas, el floema de células pequeñas, con paredes primarias de diferente espesor, y células de parénquima entre el floema y el xilema (figuras $4 \mathrm{~d}$, e); además, el periciclo está constituido por 1 a 2 estratos de células, con paredes delgadas y algunas células ocluidas por contenido obscuro. La anatomía del raquis es similar a lo descrito para el pecíolo, pero solamente presenta tres 
cordones vasculares (figura 4f), porque los más pequeños se fusionan a los de mayor tamaño.

Lámina. El patrón de venación es libre (figura 5a), la vena media y las secundarias se dividen una vez cerca del margen (figura 5b). Se observan tricomas multicelulares en ambos lados de la lámina, principalmente en la vena media y las secundarias (figura 5c). Los soros se ubican en la parte media de las venas. La lámina, en vista superficial, tiene células epidérmicas con paredes onduladas. En la cara abaxial, al mismo nivel que las otras células epidérmicas (figura 5e), se encuentran los estomas polocíticos, con sus células oclusivas rodeadas por una célula subsidiaria en forma de herradura (figura 5d). En el corte transversal, la epidermis es simple, las células de la cara abaxial son pequeñas, de forma redonda y con cutícula delgada, y en la cara adaxial son redondas de mayor tamaño, con contenido obscuro en su lumen y con una cutícula más gruesa que en la cara abaxial. El mesofilo está formado por parénquima esponjoso; sus células son de forma irregular, con espacios intercelularesamplios y abundantes cl o roplastos (figura 5e). El tejido vascular de la vena media es similar al arreglo descrito para los cordones vasculares de menor tamaño del pecíolo; sin embargo, todas las células de la banda cortical tienen un tamaño similar (figuras 5f, g). Además, en la cara adaxial de la vena media hay de 2 a 3 estratos de fibras subyacentes a la epidermis (figura $5 \mathrm{~g}$ ).

\section{Discusión}

La anatomía de la raíz, el rizoma, el pecíolo y la lámina de Ctenitis melanosticta es muy similar a la descrita para algunos géneros de la familia Dryopteridaceae y otras familias relacionadas filogenéticamente. Ogura (1972) señala que en la raíz de los helechos, el córtex puede estar constituido sólo por parénquima o por esclerénquima, o por ambos. En $C$. melanosticta se presentan los dos tipos de tejidos, como en otras especies de helechos avanzados (cuadro 1), pero difieren de las especies de Cheilanthes, en las que el córtex está totalmente formado por esclerénquima (Herrera-Monsivais, 1993). Se ha dado una interpretación diferente a las células del córtex con paredes gruesas en Thelypteris (Zavaro-Pérez et al., 1995), género para el que se interpretan como paredes primarias y en una transición entre parénquima y colénquima, o bien como un colénquima atípico. Los resultados de las pruebas con

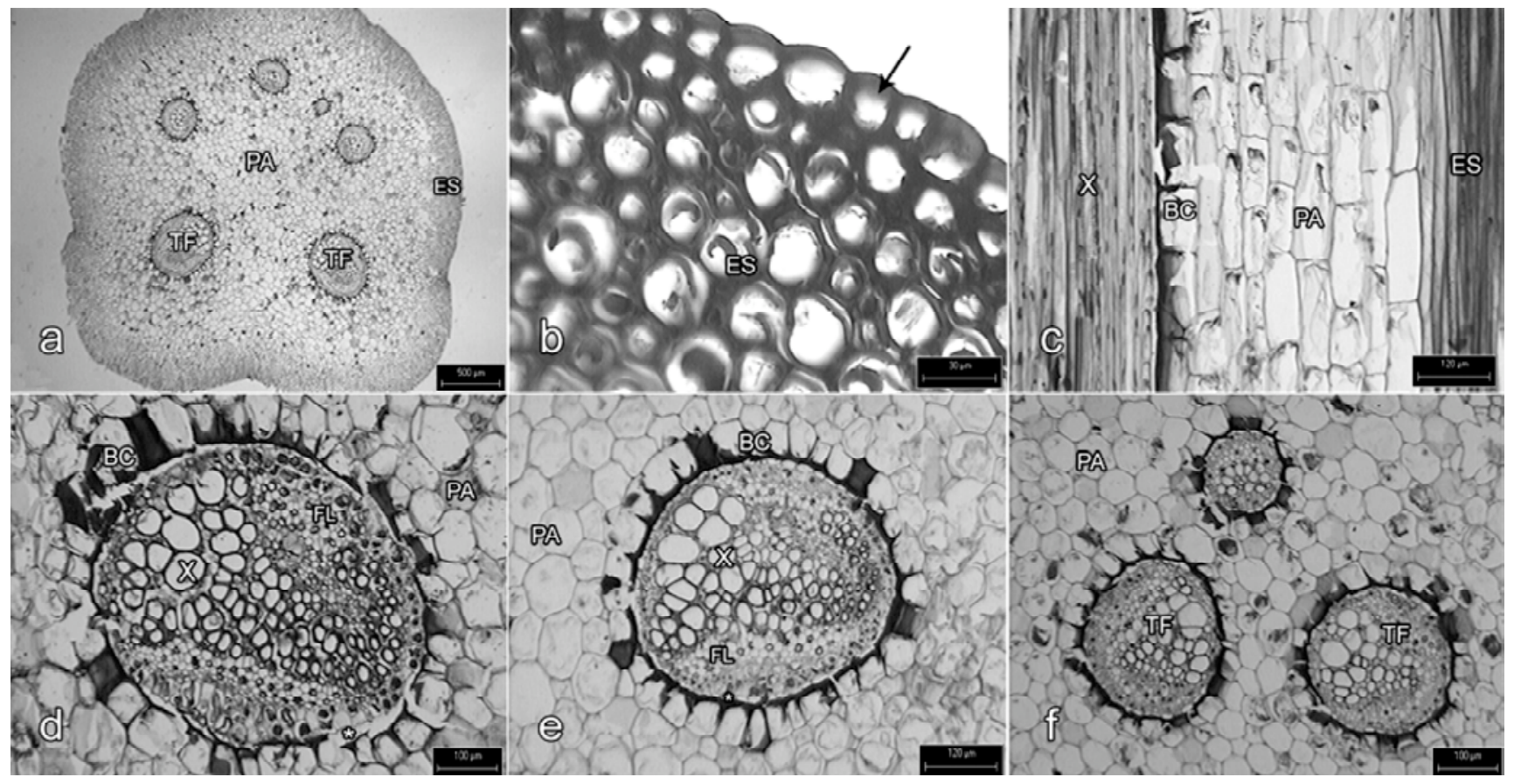

Figura 4. Pecíolo, cortes transversales (con excepción de c). a. Pecíolo cilíndrico con surco en la cara adaxial. b. Epidermis y esclerénquima. c. Corte longitudinal de cordón vascular de la traza foliar y tejido fundamental. d. Cordón vascular de la traza foliar en la base del pecíolo con arreglo del xilema en forma de hipocampo y banda cortical. e. Parte superior del pecíolo con células de la banda cortical de tamaño similar. f. Traza foliar múltiple de tres cordones vasculares en el raquis. Escalas: $\mathbf{a}=550 \mu \mathrm{m}, \mathbf{b}=30 \mu \mathrm{m}, \mathbf{d}, \mathbf{f}=100 \mu \mathrm{m}, \mathbf{c}, \mathbf{e}$ $=120 \mu \mathrm{m}$. Clave de símbolos: $*=$ endodermis, flecha $=$ epidermis, $\mathrm{BC}=$ banda cortical, $\mathrm{ES}=$ esclerénquima, $\mathrm{FL}=\mathrm{floema}, \mathrm{TF}=\mathrm{traza}$ foliar, $\mathrm{PA}=$ parénquima, $\mathrm{X}=$ xilema. 


\section{Victoria Hernández-Hernández, Teresa TerRaZas y KLAus Mehltreter}
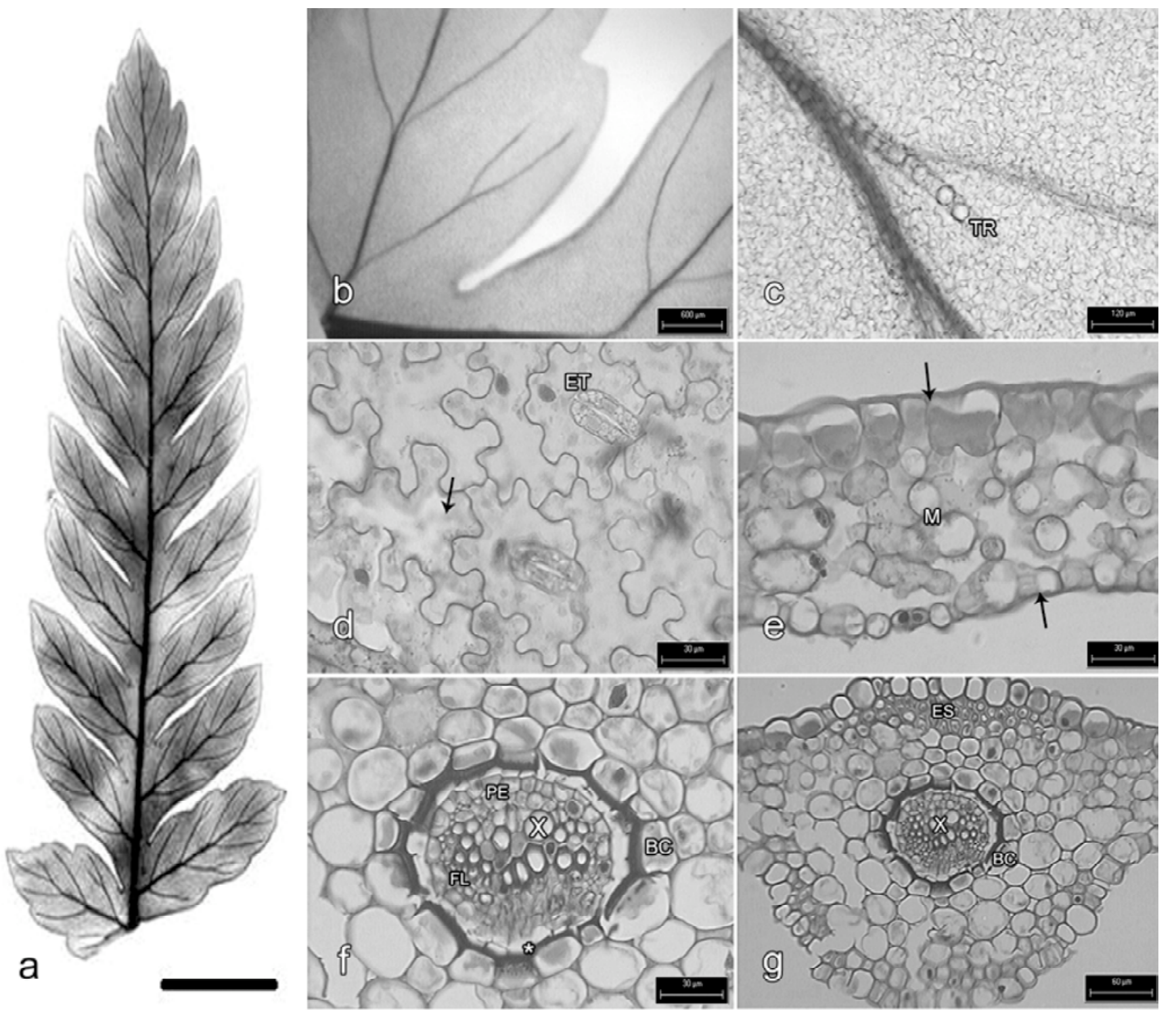

Figura 5. Lámina foliar. a-c. Diafanizada. a. Pinnula de la fronda. b. Venación libre. c. Tricoma. d. Corte para de rmal. Células epidérmicas con paredes onduladas y estomas polocíticos (fl e chas). e-g. Cortes transve rsales. e. Epidermis y mesofilo. f. Detalle tejido vascular de la vena media y banda cortical. g. Costa. Escalas: $\mathbf{a}=1 \mathrm{~cm}, \mathbf{b}=600 \mu \mathrm{m}, \mathbf{c}=120 \mu \mathrm{m}, \mathbf{d}, \mathbf{e}, \mathbf{f}=30 \mu \mathrm{m}, \mathbf{g}=60 \mu \mathrm{m}$. Clave de símbolos: $*=$ endodermis, flecha $=$ epidemis, $\mathrm{BC}=$ banda cotical, $\mathrm{ES}=$ esclerenquima, $\mathrm{ET}=$ estoma; $\mathrm{M}=$ mesofilo, $\mathrm{TR}=$ tricoma, $\mathrm{X}=\mathrm{xilema}$.

floroglucinol y ácido clorhídrico mostraron que las paredes están lignificadas y por ello se interpretan como esclerénquima en $C$. melanosticta. Además, se observó en $C$. melanosticta la presencia de hifas fungales, las cuales también se han registrado en 16 especies de helechos de Paquistán (Iqbal et al., 1981). Con respecto al tejido vascular de la raíz, se ha descrito como un haz vascular central donde el protoxilema generalmente es diarco y triarco, con modificación a monarco en Azolla y Ophioglossum y a poliarco en las familias Ophioglossaceae, Hymenophyllaceae, Gleicheniaceae y Marattiaceae (Ogura, 1972). La raíz de C. melanosticta es diarca, similar a lo registrado en
C. ursina (Sánchez-Morales et al., 2006), en otros Dryopteridaceae (White, 1970; Moran, 1987) y en los géneros Diplazium (Jarquín-Pacheco et al., 2006), Lophosoria y Sphaeropteris (Lucansky y White, 1976). La escasa información disponible actualmente sobre la anatomía de la raíz no permite hacer generalizaciones; no obstante, se observa una tendencia a presentar un arreglo diarco en los helechos más avanzados.

La anatomía del rizoma de $C$. melanosticta es muy parecida a la descrita para el género Dryopteris y de acuerdo con Bierhorst (1971) se considera avanzada en compara- 
Cuadro 1. Comparación de la anatomía de la raíz, el rizoma, el pecíolo y la lámina en diferentes géneros y especies de helechos. Abreviaturas: Raíz, $d=$ diarca, $t=$ triarca, cpe = córtex con parénquima y esclerénquima, $c d$ = córtex con esclerénquima; Rizoma, ne $=$ nidos de esclereidas, $\mathrm{bc}=$ banda cortical; Pecíolo, $\mathrm{ntf}=$ número de trazas foliares, conxil = configuración del xilema, bc = banda cortical; Lámina, meu = mesofilo unifacial, meb = mesofilo bifacial, $v m e=$ vena media con esclerénquima, $\mathrm{mvco}=$ vena media con colénquima, bc $=$ banda cortical. $\mathrm{P}=$ presencia, $\mathrm{A}=$ ausencia.

\begin{tabular}{|c|c|c|c|c|c|c|c|c|c|c|c|c|c|c|}
\hline \multirow[t]{2}{*}{ Géneros y especies } & \multicolumn{4}{|c|}{ Raíz } & \multicolumn{2}{|c|}{ Rizoma } & \multicolumn{3}{|c|}{ Pecíolo } & \multicolumn{5}{|c|}{ Lámina } \\
\hline & d & $\mathrm{t}$ & cpe & $\overline{\mathrm{ce}}$ & ne & $\overline{b c}$ & $\overline{\mathrm{ntf}}$ & conxil & $\overline{b c}$ & meu & meb & vme & mvco & bc \\
\hline Adiantum concinnum ${ }^{17}$ & & & & & & & & & A & & & & & \\
\hline Anemia phyllitidis ${ }^{17,18}$ & & & $\mathrm{P}^{18}$ & & & & & & $\mathrm{~A}^{17}$ & & & & & \\
\hline Alsophila firma ${ }^{17}$ & & & & & & & & & A & & & & & \\
\hline Asplenium $1^{2}$ & & & & & & & & & & & $\mathrm{P}$ & & $P$ & \\
\hline A. sphaerosporum ${ }^{17}$ & & & & & & & & & A & & & & & \\
\hline Athyrium ${ }^{9}$ & & & & & & & 2 & & & & & & & \\
\hline Blechnum ${ }^{4}$ & & & & & & & & Arco & & & & & & \\
\hline B. appendiculatum ${ }^{17,18}$ & & & & & & & $3^{18}$ & & $\mathrm{P}^{17}$ & & & & & $\mathrm{~A}^{18}$ \\
\hline B. schiedeanum ${ }^{17,18}$ & & & & & & & $13^{18}$ & & $\mathrm{P}^{17}$ & & & & & $\mathrm{~A}^{18}$ \\
\hline Bolbitis ${ }^{4}$ & & & & & & & & Arco & & & & & & \\
\hline B. bernoullii ${ }^{17}$ & & & & & & & & & $\mathrm{P}$ & & & & & \\
\hline Botrychium decompositum $^{17}$ & & & & & & & & & A & & & & & \\
\hline Campyloneurum angustifolium ${ }^{8}$ & $P$ & & $P$ & & $P$ & A & & & $\mathrm{P}$ & $P$ & & & & $P$ \\
\hline C. phyllitidis & $\mathrm{P}$ & & $P$ & & $\mathrm{P}$ & $A$ & & & $\mathrm{P}$ & $P$ & & & & $\mathrm{P}$ \\
\hline C. tenuipes $^{8}$ & $P$ & & $P$ & & $P$ & $\mathrm{~A}$ & & & $\mathrm{P}$ & $P$ & & & & $\mathrm{P}$ \\
\hline C. xalapense ${ }^{8}$ & $P$ & & $P$ & & $P$ & A & & & $\mathrm{P}$ & $P$ & & & & $\mathrm{P}$ \\
\hline Cheilanthes $^{10}$ & & & & $\mathrm{P}$ & & & & & & & $P$ & & & \\
\hline C. cucullans $s^{17,18}$ & & & $P^{18}$ & & & & & & $\mathrm{~A}^{17}$ & & & & & \\
\hline Ctenitis melanosticta & $\mathrm{P}$ & & $P$ & & $\mathrm{P}$ & A & 5 & Arco & $\mathrm{P}$ & $P$ & & $P$ & & $\mathrm{P}$ \\
\hline C. submarginalis ${ }^{17}$ & & & & & & & & & $\mathrm{P}$ & & & & & \\
\hline C. ursina ${ }^{16}$ & $P$ & & & & & & 5 & & & & & & & \\
\hline Cystopteris fragilis ${ }^{17}$ & & & & & & & & & $\mathrm{P}$ & & & & & \\
\hline Dennstaedtia cicutaria ${ }^{17,18}$ & & & $P^{18}$ & & & & & & $\mathrm{~A}^{17}$ & & & & & \\
\hline Diplazium accedens ${ }^{9}$ & & & & & & & & uilla o "W & & & & & & \\
\hline D. esculentum 9 & & & & & & & & uilla o " & & & & & & \\
\hline D. expansum ${ }^{17}$ & & & & & & & 2 & "V" & $\mathrm{P}$ & & & & & \\
\hline D. franconis ${ }^{17}$ & & & & & & & 2 & & $\mathrm{P}$ & & & & & \\
\hline D. kunstleri ${ }^{9}$ & & & & & & & 1 & & & & & & & \\
\hline D. polypopodioides ${ }^{9}$ & & & & & & & & uilla o "W & & & & & & \\
\hline D. striatum ${ }^{15}$ & $P$ & & & & & & & Bastón & & & & & & \\
\hline D. ternatum ${ }^{15}$ & $P$ & & & & & & & & & & & & & \\
\hline Dryopteris cinnamomea ${ }^{14}$ & & & & & $\mathrm{P}$ & A & & Arco & $\mathrm{P}$ & & $P$ & & $P$ & $\mathrm{P}$ \\
\hline D. maxonii' ${ }^{14}$ & & & & & $P$ & $\mathrm{~A}$ & $3-4$ & Arco & $\mathrm{P}$ & & $\mathrm{P}$ & & $P$ & $\mathrm{P}$ \\
\hline D. patula ${ }^{14}$ & & & & & $P$ & $\mathrm{~A}$ & & Arco & $\mathrm{P}$ & & $P$ & & $P$ & $\mathrm{P}$ \\
\hline D. rosea $^{14}$ & & & & & $P$ & A & & Arco & $\mathrm{P}$ & & $\mathrm{P}$ & & $P$ & $\mathrm{P}$ \\
\hline D. rossii ${ }^{14}$ & & & & & $P$ & A & & Arco & $\mathrm{P}$ & & $\mathrm{P}$ & & $P$ & $P$ \\
\hline D. wallichiana ${ }^{14}$ & & & & & A & $\mathrm{A}$ & $8-9$ & Arco & $\mathrm{P}$ & & $\mathrm{P}$ & & $P$ & $P$ \\
\hline Elaphoglossum ${ }^{7,17}$ & & & & & & & $2-5^{7}$ & $\mathrm{ArCO}^{17}$ & & & & & & \\
\hline E. crassipes ${ }^{7}$ & & & & & & & & & $\mathrm{P}$ & & $\mathrm{P}$ & $P$ & & \\
\hline E. gayanum ${ }^{7}$ & & & & & & & & & $\mathrm{P}$ & & $\mathrm{P}$ & $P$ & & \\
\hline E. lindbergir & & & & & & & & & $\mathrm{P}$ & $P$ & $\mathrm{P}$ & $P$ & & \\
\hline E. lorentzii & & & & & & & & & $\mathrm{P}$ & & $P$ & $P$ & & \\
\hline E. peltatum ${ }^{17,18}$ & & & $\mathrm{P}^{18}$ & & & & & & $\mathrm{P}^{17}$ & & & & & \\
\hline E. spathulatum 7 & & & & & & & & & $\mathrm{P}$ & & $\mathrm{P}$ & $P$ & & \\
\hline E. yungense & & & & & & & & & $\mathrm{P}$ & & $\mathrm{P}$ & $P$ & & \\
\hline Lomariopsis recurvata $^{17}$ & & & & & & & & & $\mathrm{P}$ & & & & & \\
\hline Lophosoria quadripinnata ${ }^{2,17}$ & $\mathrm{P}^{2}$ & & $\mathrm{P}^{2}$ & & & & & & $\mathrm{~A}^{17}$ & & & & & \\
\hline Macrothelypteris torresiana ${ }^{17}$ & & & & & & & & & $\mathrm{P}^{17}$ & & & & & \\
\hline Marattia laxa ${ }^{17,19}$ & & & & & & & & & $A$ & & $\mathrm{P}^{19}$ & & & \\
\hline Microgramma nitida ${ }^{8}$ & $\mathrm{P}$ & & $P$ & & & $\mathrm{P}$ & & & $\mathrm{P}$ & $P$ & & & & \\
\hline Nephrolepis cordifolia ${ }^{17}$ & & & & & & & & & $\mathrm{P}$ & & & & & \\
\hline
\end{tabular}


Victoria HernándeZ-Hernández, Teresa TerraZas y Klaus Mehltreter

\begin{tabular}{|c|c|c|c|c|c|c|c|c|c|c|c|c|c|c|}
\hline \multirow[t]{2}{*}{ Géneros y especies } & \multicolumn{4}{|c|}{ Raíz } & \multicolumn{2}{|c|}{ Rizoma } & \multicolumn{3}{|c|}{ Pecíolo } & \multicolumn{5}{|c|}{ Lámina } \\
\hline & $d$ & $\mathrm{t}$ & cpe & $\overline{\mathrm{ce}}$ & ne & bc & ntf & conxil & $\overline{b c}$ & meu & meb & vme & mvco & bc \\
\hline Niphidium crassifolium ${ }^{8,17,18}$ & $\mathrm{P}^{8}$ & & & & & $\mathrm{P}^{18}$ & & & $\mathrm{P}^{17}$ & $P$ & & & & \\
\hline Olfersia cervina ${ }^{17}$ & & & & & & & & & A & & & & & \\
\hline Osmunda regalis ${ }^{17}$ & & & & & & & & & A & & & & & \\
\hline Pecluma plumula ${ }^{17}$ & & & & & & & & & A & & & & & \\
\hline Pellaea ovata ${ }^{17}$ & & & & & & & & & A & & & & & \\
\hline P. ternifolia17 & & & & & & & & & A & & & & & \\
\hline Phanerophlebia remotispora $a^{17,18}$ & & & & & & & & $\operatorname{Arco}^{17}$ & $\mathrm{P}^{17}$ & & & & & $\mathrm{~A}^{18}$ \\
\hline Phlebodium areolatum ${ }^{17,18}$ & & & & & & & & & $\mathrm{P}^{17}$ & $\mathrm{P}^{18}$ & & & & \\
\hline Plagiogyria pectinata ${ }^{17,18}$ & & & & & & & & & $\mathrm{~A}^{17}$ & & & & & $\mathrm{~A}^{18}$ \\
\hline Pleopeltis angusta & $\mathrm{P}$ & & $P$ & & & A & & & $\mathrm{P}$ & & $P$ & & & \\
\hline P. conzattii ${ }^{8}$ & $P$ & & $P$ & & & A & & & $\mathrm{P}$ & & $\mathrm{P}$ & & & \\
\hline P. crassinervata ${ }^{8}$ & $\mathrm{P}$ & & $P$ & & & A & & & $\mathrm{P}$ & & $P$ & & & $P$ \\
\hline P. polylepis ${ }^{8}$ & $P$ & & $P$ & & & $\mathrm{~A}$ & & & $P$ & & $P$ & & & \\
\hline Polybotrya $^{6}$ & $P$ & & $P$ & & A & A & & Omega & A & $P$ & & & $P$ & A \\
\hline Polypodium ${ }^{17}$ & & & & & & & & Arco & & & & & & \\
\hline P. $\operatorname{arcanum}^{13}$ & & & & & & $\mathrm{P}$ & & & $P$ & $P$ & & & & \\
\hline P. californicum $13,17,18$ & & & & & $\mathrm{~A}^{18}$ & $\mathrm{P}^{13}$ & & & $\mathrm{P}^{17}$ & $\mathrm{P}^{13}$ & & & & \\
\hline P. colpodes ${ }^{13,17}$ & & & & & & $\mathrm{P}^{13}$ & & & $\mathrm{P}^{17}$ & $\mathrm{P}^{13}$ & & & & \\
\hline P. echinolepis ${ }^{13}$ & & & & & & & & & & & $\mathrm{P}$ & & & \\
\hline P. fraternum ${ }^{13}$ & & & & & & & & & & & $\mathrm{P}$ & & & $P$ \\
\hline P. hispidulum ${ }^{18}$ & & & & & & & & & & $P$ & & & & \\
\hline P. loriceum ${ }^{13}$ & & & & & & & & & & $P$ & & & & \\
\hline P. plebeium ${ }^{17,18}$ & & & $\mathrm{P}^{18}$ & & & & & & $\mathrm{P}^{17}$ & $\mathrm{P}^{18}$ & & & & $\mathrm{P}^{18}$ \\
\hline P. plesiosorum ${ }^{13,17}$ & & & & & & & & & $\mathrm{P}^{17}$ & $\mathrm{P}^{13}$ & & & & \\
\hline P. rhachipterygium ${ }^{13,17,18}$ & & & & & $\mathrm{~A}^{18}$ & $\mathrm{~A}^{18}$ & & & $\mathrm{P}^{17}$ & $\mathrm{P}^{13}$ & & & & \\
\hline P. rhodopleurum ${ }^{13,18}$ & & & & & & $\mathrm{P}^{13}$ & & & $\mathrm{P}^{17}$ & $\mathrm{P}^{13}$ & & & & $\mathrm{P}^{18}$ \\
\hline P. subpetiolatum ${ }^{17}$ & & & & & & & & & $\mathrm{P}$ & & $P$ & & & \\
\hline P. triseriale $e^{13}$ & & & & & & & & & & & $P$ & & & \\
\hline Polystichum hartwegii' & & & $\mathrm{P}^{18}$ & & & & & Arco & $\mathrm{P}^{17}$ & & & & & \\
\hline Pteridium feei $i^{17,18}$ & & & & & & & & & $\mathrm{~A}^{17}$ & & & & & $\mathrm{~A}^{18}$ \\
\hline Pteris quadriaurita ${ }^{17,18}$ & & & $\mathrm{P}^{18}$ & & & & & & $\mathrm{~A}^{17}$ & & & & & $\mathrm{~A}^{18}$ \\
\hline Sphaeropteris elongata ${ }^{2}$ & $P$ & & $P$ & & & & & & & & & & & \\
\hline Sticherus bifidus ${ }^{17}$ & & & & & & & & & $\mathrm{~A}^{17}$ & & & & & $\mathrm{~A}^{18}$ \\
\hline Tectaria decurrens ${ }^{1}$ & $P$ & & & & & & & & & & & & & \\
\hline T. devexa' & $P$ & & & & & & & & & & & & & \\
\hline T. fernandensis ${ }^{1}$ & $\mathrm{P}$ & & & & & & & & & & & & & \\
\hline T. heracleifolia', 5, 17,18 & $P^{1}$ & & & & & & $6^{5}$ & $\operatorname{Arco}^{17}$ & $\mathrm{P}^{17}$ & $\mathrm{P}^{5}$ & & & & $\mathrm{P}^{18}$ \\
\hline T. incisa ${ }^{1}$ & $P$ & & & & & & & & & & & & & \\
\hline T. mexicana ${ }^{5}$ & & & & & & & 8 & & & $P$ & & & & \\
\hline T. polymorpha' & $P$ & & & & & & & & & & & & & \\
\hline Terpsichore asplenifolia' ${ }^{17}$ & & & & & & & & & A & & & & & \\
\hline Thelypteris ${ }^{11}$ & & & & & & & 2 & & & & & $P$ & & \\
\hline T. linkiana ${ }^{17,18}$ & & & & & & & $2^{17}$ & & $\mathrm{~A}^{17}$ & & & & & $\mathrm{~A}^{18}$ \\
\hline T. rudis $^{17,18}$ & & & & & & & $2^{17}$ & & $\mathrm{P}^{17}$ & & & & & $\mathrm{~A}^{18}$ \\
\hline Trichomanes capillaceum ${ }^{17,18}$ & & & & & & & & & $\mathrm{~A}^{17}$ & & & & & $\mathrm{~A}^{18}$ \\
\hline Vittaria graminifolia ${ }^{17}$ & & & & & & & & & A & & & & & \\
\hline Woodsia mollis ${ }^{17}$ & & & & & & & 2 & & A & & & & & \\
\hline Woodwardia areolata ${ }^{3}$ & $P$ & & $P$ & & A & & 6 & & $\mathrm{P}$ & & $P$ & & & $P$ \\
\hline W. radicans ${ }^{3}$ & & $\mathrm{P}$ & $P$ & & A & & 7 & & $\mathrm{P}$ & & $P$ & & & $P$ \\
\hline W. virginica ${ }^{3}$ & $P$ & & $\mathrm{P}$ & & A & & $7-8$ & & $P$ & & $P$ & & & $P$ \\
\hline W. $\times$ semicordata $^{17,18}$ & & & $\mathrm{P}^{18}$ & & & & $3^{18}$ & $\operatorname{Arco}^{17}$ & $\mathrm{P}^{17}$ & & & & & \\
\hline
\end{tabular}

Fuentes: 'White (1970); ${ }^{2}$ Lucansky y White (1976); ${ }^{3}$ Lucansky (1981); ${ }^{4}$ Ogura (1982); ${ }^{5}$ Velásquez-Montes (1983); ${ }^{6}$ Moran (1987); ${ }^{7}$ Guantay y Hernández (1990); ${ }^{8} Z$ lotnik-Espinosa (1991); ${ }^{9}$ Umikalsom (1992); ${ }^{10}$ Herrera-Monsivais (1993); "Zavaro-Pérez et al. (1995); ${ }^{12} \mathrm{Chaerle}$ y Viane

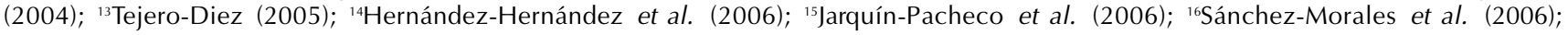
${ }^{17}$ Hernández-Hernández et al. (en prensa); ${ }^{18}$ Hernández-Hernández (datos no publicados); ${ }^{19}$ Velasco-Colín (1971). 
ción con otros géneros. Sin embargo, los nidos de esclereidas de $C$. melanosticta difieren de los de Dryopteris patula por la ausencia de espacios intercelulares, y de los de $D$. rossii y $D$. maxonii por la falta de cristales en la periferia de los nidos (Hernández-Hernández et al., 2006). También se han reportado nidos de esclereidas en otros géneros de Dryopteridaceae (Moran, 1987), en Campyloneurum de la familia Polypodiaceae (Zlotnik-Espinosa, 1991) y en algunos miembros de la familia Lomariopsidaceae (Moran, 1987). Al contrario, no se han observado nidos de esclereidas en Polybotrya (Moran, 1987), Polypodium (HernándezHernández, datos no publicados) y Woodwardia (Lucansky, 1981), por lo que se considera un rasgo variable a nivel de familia y su valor diagnóstico deberá usarse con precaución, hasta que un mayor número de géneros haya sido estudiado.

Algunos autores han reportado la presencia de la banda cortical en el rizoma de Microgramma nitida, Niphidium crassifolium y varias especies de Polypodium (ZlotnikEspinosa, 1991; Tejero-Diez, 2005). Sin embargo, en el rizoma de la especie en estudio, C. melanosticta, y de especies de los género Dryopteris, Polybotrya, Campyloneurum, Pleopeltis y Polypodium rhachiptery gium, la banda cortical no se diferencia (Moran, 1987; Zlotnik-Espinosa, 1991; Hernández-Hemández et al., 2006; en prensa).

El número, la forma y el arreglo de los cordones vasculares de la traza foliar del pecíolo se consideran caracteres informativos para la identificación de las familias de helechos (Lin y DeVol, 1977, 1978). El pecíolo de Ctenitis melanosticta tiene una traza foliar múltiple con cinco cordones vasculares en la base del pecíolo y tres en el raquis, al igual que C. ursina (Sánchez-Morales et al., 2006). Esta variación acrópeta en el número de cordones vasculares también se presenta en otras especies de Blechnum, Diplazium, Dryopteris, Elaphoglossum y Woodwardia (cuadro 1). Los géneros Athyrium, Diplazium y Thelypteris se caracterizan por tener dos cordones vasculares en la traza foliar en la base del pecíolo y hacia el raquis se fusionan en una; la excepción es Diplazium kunstleri, con una traza foliar formada por un único cordón vascular a lo largo del pecíolo (Umikalsom, 1992; Zavaro-Pérez et al., 1995). La presencia de una traza foliar múltiple $C$. melanosticta es un atributo que se considera avanzado en comparación con los géneros de otras familias de helechos (Bierhorst, 1971; Ogura, 1972). Además, el arreglo de las trazas foliares en C. melanosticta es en forma de arco, con dos trazas de mayor tamaño hacia la cara adaxial y el resto de menor tamaño hacia la cara abaxial; esta disposición es similar a la registrada en los géneros Bolbitis, Blechnum, Dryopteris, Elaphoglossum, Phanerophlebia, Polystichum, Tectaria y Woodwardia (Ogura, 1972; Hernández-Hernández et al., 2006, en prensa). El género Polybotrya (Dryopteridaceae) difiere en el arreglo del xilema de la traza foliar en forma de omega (Moran, 1987), mientras que en Diplazium la configuración del xilema se presenta en forma de quilla o "W" (Umikalsom, 1992), en hipocampo o en bastón (Jarquín-Pacheco et al., 2006), o en "V" y con la terminación del xilema arqueada hacia adentro (HernándezHernández, datos no publicados). Aunque la banda cortical no se diferencia en el rizoma, ésta está presente en el pecíolo de $C$. melanosticta, al igual que en especies de 20 géneros de familias consideradas avanzadas (Lucansky, 1981, Guantay y Hemández, 1990; Zlotnik-Espinosa, 1991; Tejero-Diez, 2005; Hernández-Hernández et al., en prensa), pero está ausente en otros taxa (cuadro 1). En Dryopteris se presentan los mismos engrosamientos de las paredes de la banda cortical en forma de "U" y contenido obscuro en sus lúmenes celulares (Hernández-Hernández et al., 2006) que en $C$. melanosticta, pero difieren por el menor tamaño de las células hacia el centro de los mayores cordones vasculares de la traza foliar múltiple. Sin embargo, observaciones preliminares de Ctenitis submarginalis muestran que las células de la banda cortical tienen un tamaño similar a lo largo de todo el pecíolo (HernándezHernández, datos no publicados). La presencia de la banda cortical en los cordones vasculares de la traza foliar múltiple se interpreta como un carácter avanzado en $C$. melanos ticta y los miembros de las familias Blechnaceae, Dryopteridaceae, Lomariopsidaceae, Nephrolepidaceae, Polypodiaceae y Thelypteridaceae (Hernández-Hernández et al., en prensa).

La venación de $C$. melanosticta es libre, con la venas secundarias divididas una vez cerca del margen, a diferencia de Dryopteris, cuyas venas secundarias se bifurcan dos o tres veces (Hemández-Hemández et al., 2006). En Polybotrya y Polypodium la venación puede ser libre o anastomosada (Moran, 1987); en el último género las areolas presentan una forma diferente (Tejero-Diez, 2005). De acuerdo con la propuesta de Bierhorst (1971), la venación dicotómica es un rasgo primitivo y esta interpretación se da a la venación de $C$. melanosticta. Las células del mesofilo fuertemente lobuladas de Ctenitis melanosticta son similares a las descritas para especies de los géneros Campyloneurum, Elaphoglossum, Microgramma, Niphidium, Phlebodium, Polybotrya, Polypodium y Tectaria (Velásquez-Montes, 1983; Moran, 1987; Guantay y Hernández, 1990; Zlotnik-Espinosa, 1991; Tejero-Diez, 2005). En otros géneros (cuadro 1) se reporta un mesofilo bifacial, con los parénquimas en empalizada y esponjoso diferenciados. En los géneros Elaphoglossum y Polypodium se han registrado especies con mesofilo uni y bifacial. Estos géneros se distinguen por la amplia diversidad de ambientes y hábitats en los cuales se encuentran, desde selvas y bosque mesófilo de montaña hasta encinar y pinar; además las plantas pueden ser terrestres, epipétricas y epifitas (Mickel y Beitel, 1988; Moran, 1995). Ctenitis melanosticta presenta en el lado adaxial de la vena media 
varios estratos de fibras subyacentes a la epidermis, similar a lo descrito en Elaphoglossum (Guantay y Hernández, 1990) y en Thelypteris (Zavaro-Pérez et al., 1995). Para otros géneros como Asplenium, Dryopteris y Polybotrya se registra colénquima en la cara abaxial de la vena media (cuadro 1). Además, Chaerle y Viane (2004) mencionan la presencia de una hipodermis colenquimatosa en ambos lados de la lámina en Asplenium, que se conecta con el colénquima de la costa y las cóstulas, formando las venas falsas. Es interesante notar que las fibras del esclerénquima solamente se presentan en la lámina y en el pecíolo, y seguramente tienen una función mecánica para darle ri gidez — p e ro también flexibilidad — a la fronda, mientras que la presencia de escle reidas en la raíz y el ri zoma se relaciona con una función de protección hacia el tejido vascular.

El haz vascular de la vena media está rodeado por la banda cortical similar a lo descrito para Campyloneurum, Dryopteris, Pleopeltis, Polypodium y Tectaria (HernándezHernández et al., 2006; Hernández-Hernández, datos no publicados), pero no ha sido observado en las especies estudiadas de Blechnum, Phanerophlebia, Plagiogyria, Polybotrya y Thelypteris (Moran, 1987; HernándezHernández, datos no publicados), por lo que podría ser un carácter con valor diagnóstico. Con la finalidad de confirmar la importancia de la banda en la lámina están siendo estudiadas otras especies de Dryopteridaceae.

En conclusión, Ctenitis melanosticta comparte rasgos anatómicos de sus diferentes órganos con otros helechos considerados avanzados, como los pertenecientes a la familia Dryopteridaceae. Entre los caracteres anatómicos que $C$. melanosticta comparte con este grupo de helechos están la raíz diarca, el córtex formado por parénquima y esclerénquima (esclereidas y nidos de esclereidas) en el rizoma, la presencia de banda cortical en pecíolo y lámina, así como el mesofilo unifacial y las fibras subyacentes a la epidermis de la vena media en la lámina.

\section{Agradecimientos}

La primera autora agradece al CONACYT la beca para realizar estudios de doctorado (162688), a José Luis González G. y Adriana Hernández Rojas por la ayuda en la recolecta de material, a Nubia Orozco Ospina y Alexandre Salino por la revisión critica al manuscrito y a Jorge Meave por sus atinadas sugerencias editoriales.

\section{Literatura citada}

Bierhorst D.W. 1971. Morphology of Vascular Plant. MacMillan, Nueva York.

Chaerle P. y Viane R.L. 2004. Leaf anatomy and the ocurrence of false veins in Asplenium (Aspleniaceae, Pterydophyta). Botanical Journal of the Linnean Society 145:187-194.

Crabbe J.A., Jermy A.C. y Mickel J.T. 1975. A new generic sequence for the Pteridophyte herbarium. Fern Gazette 11:141162.

Guantay M.E. y Hernández M.T. 1990. Estudio morfoanatómico de las especies del género Elaphoglossum Schott de Tucumán. Lilloa 38:73-84.

Hasebe M., Wolf P.G., Pryer K.M., Ueda K., Sano R.M.I., Gastony G.J., Yokoyama J., Manhart J.R., Murakami N., Crane E.H., Haufler C.H. y Hauk W.D. 1995. Fern phylogeny based on $r b c \mathrm{~L}$ nucleotide sequences. American Fern Journal 85:134181.

Hernández-Hemández V., Te rrazas T. y Ángeles G. 2006. Anatomía de seis especies de Dryopteris en México. Revista de Biología Tropical 54:1157-1169.

Hernández-Hernández V., Terrazas T., Mehltreter K. y Ángeles G. En prensa. Systematic survey of the cortical band and its relationship with foliar trace configurations in Pteridophyte petioles. Systematic Botany.

Hernández-Rojas A.C. 2006. Fenología de helechos terrestres en un fragmento de bosque mesófilo de montaña en Xalapa, Veracruz, México. Tesis de Licenciatura, Facultad de Biología, Universidad Veracruzana, Xalapa, 69 pp.

Herrera-Monsiváis C.M. 1993. Anatomía de dos especies de Cheilanthes Swartz. Tesis de Maestría, Colegio de Postgraduados, Montecillo, Edo. de México, 107 pp.

Iqbal S.H., Yousaf M. y Younus M. 1981. A field survey of mycorrhizal associations in ferns of Pakistan. New Phytologist 87:69-79.

Jarquín-Pa checo M.B., Pa checo L., Quintanar-Isaías P.A. y Sánchez-Morales A. 2006. Anatomía e histoquímica del esporofito de Diplazium striatum (L.) Presl y Diplazium ternatum Liebm. (Athyriaceae. Pteridophyta). Libro de Resúmenes IX Congreso Latinoamericano de Botánica, Santo Domingo.

Lin B.L. y DeVol C.D. 1977. The use of stipe characters in fern taxonomy I. Taiwania 22:91-99.

Lin B.L. y DeVol C.D. 1978. The use of stipe characters in fern taxonomy II. Taiwania 23:77-95.

Lucansky T.W. 1981. Chain ferns of Florida. American Fern Journal 71:101-108.

Lucansky T.W y White R.A. 1976. Comparative ontogenetic studies in young sporophytes of tree ferns. I. A primitive and an advanced taxon. American Journal of Botany 63:463-472.

Mickel J.T. y Beitel J.M. 1988. Pteridophyte flora of Oaxaca, Mexico. Memoirs of the New York Botanical Garden 46:320327.

Mickel J.T. y Smith A.R. 2004. The Pteridophytes of Mexico. Memoirs of the New York Botanical Garden 88:1-1054.

Miranda F. y Hernández-X. E. 1963. Los tipos de vegetación de México y su clasificación. Boletín de la Sociedad Botánica de México 28:29-72.

Moran R.C. 1987. Monograph of the neotropical fern genus Polybotrya (Dryopteridaceae). Illinois Natural History Survey Bulletin 34:1-138.

Moran R.C. 1995. Ctenitis. En: Davidse G., Sousa M. y Knapp S. Eds. Flora Mesoamericana. Psilotaceae a Salviniaceae, Vol. 1, pp. 196-200, Universidad Nacional Autónoma de México, Missouri Botanical Garden y The Natural History Museum, México, D.F.

Ogura Y. 1972. Comparative Anatomy of the Vegetative Organs of the Pteridophytes. Borntraeger, Berlín.

Ruzin S.E. 1999. Plant Microtechnique and Microscopy. Oxford 
University Press, Nueva York.

Sánchez-Morales A., Pacheco L., Jarquín-Pacheco M.B. y Quintanar-Isaías P.A. 2006. Anatomía del esporofito de Ctenitis ursina (Tectariaceae, Pteridophyta). Libro de Resúmenes IX Congreso Latinoamericano de Botánica, Santo Domingo.

Schmid R. 1982. The terminology and classification of steles: Historical perspective and the outlines of a system. The Botanical Review 4:817-931.

Smith A.R. 1981. Pteridophytes. En: Breedlove D.C. Ed. Flora de Chiapas, Vol. 2, pp. 1-370, California Academy of Science, San Francisco.

Tejero-Diez J.D. 2005. Revisión taxonómica del complejo Polypodium plesiosorum Kunze (Polypodiaceae, Polypodiophyta). Tesis de Doctorado, Universidad Autónoma Metropolitana-Iztapalapa, México, D.F., 115 pp.

Tryon R.M. y Tryon A.F. 1982. Ferns and Allied Plants with Special Reference to Tropical America. Springer-Verlag, Nueva York.

Umikalsom Y. 1992. Anatomical studies of the Malaysian
Aspleniaceae and Athyriaceae. Botanical Journal of the Linnean Society 110:111-119.

Velasco-Colín R. 1971. Estudio anatómico comparativo de dos especies del género Marattia (Marattiales, Pteridophyta). Tesis de Licenciatura, Universidad Nacional Autónoma de México, México, D.F., 63 pp.

Velásquez-Montes F.E. 1983. Anatomía comparada de dos especies del género Tectaria Cav. (Filicales, Pteridophyta). Tesis de Licenciatura, Universidad Nacional Autónoma de México, México, D.F., 62 pp.

White R.A. 1970. Comparative anatomy of the Aspidiaceae. I. Stelar pattern development in young sporophytes of Tectaria. American Journal of Botany 57:458-467.

Zavaro-Pérez C.A., Sánchez-Villaverde C. y Bozán J. 1995. Anatomía de las especies cubanas del género Thelypteris Schmidel, subgénero Cyclosorus Morton. Fontqueria 42:195198.

Zlotnik-Espinosa A. 1991. Anatomía de Polypodiaceae s. str. Tesis de Maestría, Universidad Nacional Autónoma de México, México, D.F., 103 pp.

Recibido: 23 de octubre de 2006

Versión corregida: $1^{\circ}$ de febrero de 2007

Aceptado: $1^{\circ}$ de febrero de 2007 